\title{
LA ECONOMÍA EN LOS PROGRAMAS DE NEGOCIOS ${ }^{1}$
}

\section{ECONOMICS IN BUSINESS PROGRAMS}

Ricardo Chica.

Director CEA UTB-UAM

Colombia

Palabras Clave: Programas de negocios, teoría económica, agentes económicos

Keyword: business programs, economic theory, economic agentss.

\section{RESUMEN}

La necesidad de que los estudiantes de los programas de negocios tengan entrenamiento en teoría económica esta basada en la importancia de que ganen un entendimiento de cuatro conceptos básicos: 1) la conducta racional de los agentes económicos; 2) su comportamiento estratégico; 3) los contraints que ellos enfrentan; 4) los trade-offs que estos constraints les imponen. Todos estos principios adquieren una nueva dimensión al considerar los negocios internacionales. Después de considerar estos aspectos se introducirá una breve referencia a unas áreas de overlapping o interacción entre la economía y los negocios. Finalmente se concluirá enfatizando la importancia de articular el abordamiento de diversos problemas en una discusión integrada en la perspectiva del Desarrollo Económico como forma de implementar la idea central del documento de entrenar en el abordamiento y la solución de problemas empresariales desde una perspectiva económica. 


\section{ABSTRACT}

This paper attempts to show the need that students in business programs have about training in economics theory based on the importance of having them approach an understanding of four basic concepts: 1) a rational behavior of economic agents; 2) their strategic behavior; 3) their constraints, and 4) the trade-offs imposed by their constraints. These principles will acquire a new dimension when considering international business. Based on these aspects, there will be a short reference to some areas which overlap or interact between economics and business. Finally, the conclusions of this paper will make emphasis on the importance of articulating the approach to various problems in an integrated discussion in respect to Economic Development as a way to implement the main idea expressed here, which is to propose the training of the approach and business problem-solving from an economic perspective.

\section{Conceptos económicos básicos}

$\mathrm{El}$ argumento sobre la necesidad de equipar a los estudiantes de negocios con conceptos económicos es una aplicación del principio de que la educación no consiste fundamentalmente en trasmisión de contenidos sino en entrenamiento en el abordamiento y la resolución de problemas. En esta dirección se trata de que el estudiante de negocios esté en capacidad de abordar los problemas empresariales desde una perspectiva económica. Lo cual redunda no solo en su capacidad para entender el contexto económico y de política económica en el que implementa sus estrategias empresariales, sino en una capacidad de entender la lógica económica de estas estrategias.

1) Al desarrollar todo su andamiaje analítico a partir del principio de la racionalidad económica individual, la teoría económica explicita en forma conceptualmente clara las implicaciones de este individualismo metodológico. La expresión de éste en los análisis microeconómicos de optimización restringida constituye una herramienta que le permite al estudiante abordar en forma rigurosa el análisis de la conducta de empresarios y consumidores ubicados en las estructuras económicas (mercados y su variedad de estructuras) desde las cuales responden a incentivos (instituciones y políticas).

2) Esta respuesta toma la forma de una interacción estratégica con otros agentes con los que se relaciona o bien competitivamente o bien

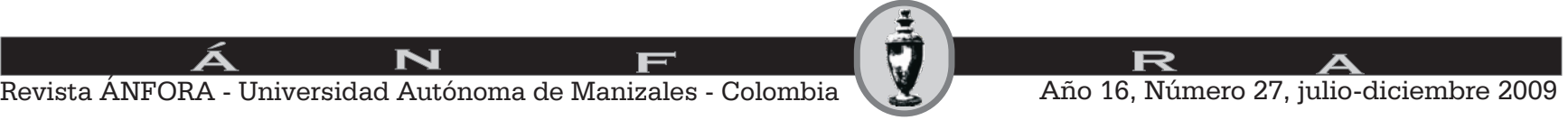


complementariamente al interior de esas estructuras. Desarrollos recientes en Organización Industrial (aplicando teoría de juegos) permiten visualizar en forma conceptualmente clara estos procesos de interacción y los resultados a los que conducen las diversas estrategias adoptadas por los agentes. De particular importancia en este análisis es la posibilidad de que soluciones cooperativas conduzcan a los jugadores a situaciones superiores a las que conduciría una estrategia no cooperativa.

3) La metodología de optimización restringida provee un prototipo a la situación enfrentada por los agentes económicos de enfrentar constraints en la búsqueda de sus objetivos estratégicos. Especialmente relevante es el hecho de que solo adquiriendo activos productivos (inversión) puede mejorar su situación futura y que normalmente las restricciones que enfrenta implican que esta inversión requiera intercambiar consumo futuro por consumo presente (obteniendo financiación que implica compromisos futuros de repago). Este principio, así como lo que se conoce como restricción intertemporal, el enunciado que afirma que la sumatoria de los VP del flujo de consumo adelantado (exceso del gasto sobre el ingreso) en cada periodo y el comprometido en el futuro como pago de las consiguientes obligaciones (el simétrico ahorro o exceso del ingreso sobre el gasto) sea cero, proveen un marco básico para analizar la dinámica tanto de agentes como de sus agregados. Considerando los agregados nacionales, este principio implica que una economía puede financiar excesos del gasto sobre el ingreso (que macroeconomicamente encuentran expresión en la ecuación (I$\mathrm{S})=(\mathrm{M}-\mathrm{X}))$ solamente mientras sus acreedores consideren que el incremento futuro del ingreso que se logra mediante el financiamiento de estos desequilibrios le va a posibilitar el repago futuro de las consiguientes obligaciones.

4) Al clarificar los constraints que restringen las conductas de los agentes y las estrategias que estos pueden implementar sujetos a estos constraints la economía explicita los trade-offs que gobiernan estas conductas. El principio there is no free lunch resume esta idea fundamental de que toda acción dirigida a un objetivo tiene un costo, que lo que el agente gane en una dirección siempre lleva involucrada una perdida en otra. Al precisar la naturaleza (forma matemática) de estas relaciones la economía permite, por una parte, la aplicación de su principio de optimización en el margen que afirma que el optimo en este trade-off se obtendrá en el punto en que lo que se gana con una

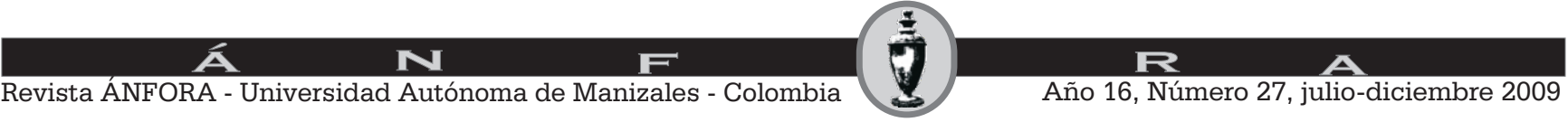


adición en una dirección es equivalente (tiene el mismo valor) que lo que se pierde en la otra; y por otra el grado de severidad del trade-off y las situaciones en que (y la forma como éste) se puede suavizar (o eliminar).

Al pasar al nivel social se puede apreciar la enorme importancia del análisis económico de la variedad de opciones enfrentadas por los agentes. Esto es así en dos direcciones fundamentales: i) la política económica; ii) y el trade off entre el beneficio individual y el beneficio colectivo. La aplicación al nivel i) de política económica se manifiesta en la imposibilidad de alcanzar simultáneamente diversos objetivos de política al nivel que seria deseable y la necesidad de apreciar los trade-offs entre ellos (los costos en términos de uno que impone la obtención de otro, lo que en términos prácticos de asignación presupuestal se traduce en la igualación de los beneficios del gasto en cada uno de ellos en el margen) y la complejidad de la relación entre instrumentos y objetivos.

La posibilidad enunciada en ii) de que el beneficio individual resulte competitivo y no complementario con el beneficio colectivo introduce la crucial dimensión social sin cuya consideración el andamiaje conceptual del estudiante de negocios se queda al nivel del principio de la mano invisible de Adam Smith (identificando el beneficio individual con el colectivo). Las varias teorías modernas de la falla de mercado muestran como éste falla como mecanismo de coordinación social y como se dan muy numerosas y relevantes situaciones en las cuales no es cierto que él traduce los esfuerzos de los individuos por su bienestar personal en bienestar colectivo. Precisar las condiciones en las cuales esto es cierto, o no lo es, le proporciona al estudiante una puerta de entrada rigurosa a la consideración de la dimensión social de la actividad empresarial, consideración en la cual conceptos como el de externalidades (y las consiguientes divergencias entre rentabilidad privada y rentabilidad social) resultan fundamentales. Solo así el estudiante podrá entender aspectos tan fundamentales como el conflicto de intereses entre el sector financiero y el sector real que se referirán mas adelante (y la falla de coordinación entre ellos a la salida de una crisis, falla que hace que cada cual buscando su propio interés inhiba la superación de ésta); y la necesidad de compensar la falla del mercado que lleva a agentes (innovadores tecnológicos y comerciales; instituciones financieras acopiando información sobre proyectos de inversión, evaluando su rentabilidad ajustada por riesgo y monitoreando su marcha) que, guiados por la racionalidad económica privada, no invierten el nivel socialmente optimo en bienes con dimensiones de bien publico. De 
manera que resulta necesario compensar la deficiencia (desde el punto de vista social) de la iniciativa privada empresarial mediante la acción del agente coordinador estatal o del regulador que genere los incentivos que compensen, en el primer caso la falla de coordinación; y en el segundo la tendencia a subinvertir en bienes con beneficios sociales superiores a sus privados (resultantes de las externalidades que generan).

La consideración del agregado nacional y de sus relaciones internacionales refuerzan la importancia de los principios económicos considerados. El tradeoff expresado en la restricción intertemporal permite entender las enormes dificultades implicadas en el proceso de desarrollo económico en tanto hecho posible por la acumulación de capital. Entender las leyes de hierro que gobiernan la dinámica del desarrollo capitalista permite entender porqué los requerimientos de ese proceso son tan difíciles de llenar y cómo, aunque la actividad empresarial juegue un papel fundamental en él, se dan numerosas situaciones en que mecanismos de coordinación institucional y gubernamental (adicionales al mercado) son necesarios. En particular, el que los procesos de acumulación de capacidades tecnoproductivas estén sujetos a rendimientos crecientes implica que la ausencia de instrumentos y estrategias colectivos se traducen en rezagos también crecientes frente a aglomeraciones que logran mediante su implementación hacer la transición de círculos viciosos de estancamiento a círculos virtuosos de crecimiento.

El que la integración de los mercados nacionales y locales en los globales haya adquirido con la globalización un papel determinante trae consigo la necesidad de considerar los procesos característicos de ella en la perspectiva de, por una parte el comportamiento estratégico de sus agentes fundamentales; y por otra, las dinámicas que gobiernan sus componentes comercial/productivo, financiero y tecnológico. Dada la masiva importancia que han adquirido las exportaciones, la IED y la organización y gestión de SPII y de CGV por parte de las MN, resulta esencial analizar sus comportamientos estratégicos para entender la dinámica de la globalización en sus tres dimensiones. Lo propio sucede con agentes críticos en las dimensión financiera (Bancos, Bancos y Fondos de Inversión, etc.) y tecnológica (sistemas de innovación). Similarmente, entender la lógica de causación acumulativa (rendimientos crecientes) que gobierna la globalización comercial/productiva, financiera y tecnológica es esencial para comprender tanto los constraints enfrentados para

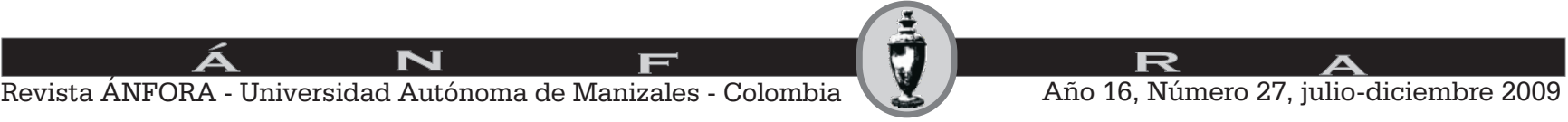


integrarse en ellas como las condiciones y mecanismos que hacen posible esta integración.

\section{2. Áreas de overlaping e interacción entre la economía y los negocios.}

Puesto el proceso de acumulación de capital al centro del análisis, a cualquier nivel de agregación (desde el agente individual hasta la economía global pasando por las aglomeraciones locales), tres áreas adquieren particular importancia para la cross-fertilization entre la economía y los negocios: 1) La estrategia empresarial; y sus condiciones 2) financieras y 3) tecnológicas.

1) La dimensión estratégica: la relación entre instrumentos y objetivos y la interacción con otros agentes son tanto destacadas como precisadas por los análisis de la organización industrial y su extensión a los análisis de la política económica domestica e internacional. La creación de ventajas competitivas para cualquier nivel de agregación procede mediante movimientos estratégicos que constituyen formas de inversión en capacidades que generan una restricción a la competencia (constituye una estructura no competitiva, en el sentido económico de competencia perfecta, tal como una barrera a la entrada). El esfuerzo por fortalecer la competitividad como condición del crecimiento está sin embargo sujeto a restricciones como la enunciada en la ecuación de precios $\mathbf{p}=(\mathbf{1}+\mathbf{m}) \mathbf{l w}$, que muestra como la única forma para fortalecer la competitividad en precios (reducir $\mathrm{p}$ ) sin deteriorar ni la capacidad de acumulación de los empresarios (el margen m) ni lo salarios de los trabajadores $(\mathrm{w})$ es incrementando la productividad (el inverso del coeficiente técnico de trabajo 1 que mide los requerimientos de trabajo por unidad de producto). Tanto los modelos de movimientos estratégicos de la organización industrial, en los que un jugador constituye una barrera a la entrada al irse adelante de los demás jugadores con la implementación de esa estrategia, como esta sencilla interpretación de la ecuación de precios como la ley de hierro de la competitividad, ilustran cómo el análisis económico provee un marco conceptual para el abordamiento de los problemas de la estrategia empresarial.

Un caso especialmente importante de aplicación de conceptos económicos es desde luego la teoría del comercio internacional, particularmente la teoría estratégica del comercio que, desde una perspectiva de rendimientos crecientes y competencia imperfecta, ha extendido los desarrollos de la

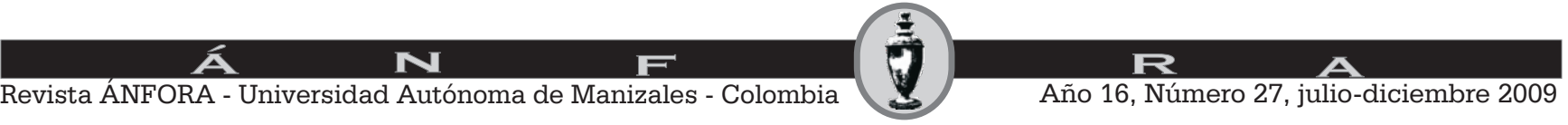


organización industrial a la política comercial. Se trata de un desarrollo que, desde una aproximación económica al problema empresarial de la competitividad, permite plantear el problema económico de esa política en una perspectiva empresarial (estratégica).

2) La dimensión financiera. El papel crucial de la financiación en el crecimiento económico, por ser condición determinante de la actividad inversora, es destacado en varias vertientes del análisis económico. Es cierto que cuerpos importantes de la teoría económica que abstraen de la falla de mercado causada por factores tan cruciales como la información asimétrica (tales como los análisis de la economía financiera que extienden los del equilibrio general competitivo a la interacción de conductas optimizantes individuales que involucran los activos financieros y los análisis tradicionales del equilibrio de la firma) no permiten apreciar adecuadamente el papel crucial de la financiación en la actividad económica; ni tampoco las complejidades del role del sistema financiero en ese proceso de crecimiento. Sin embargo, otros desarrollos (como los neokeynesianos) que si ponen al centro del análisis esas falla y asimetrías permiten, en un sentido, comprender ese papel, role y complejidades; y en otro, cómo instrumentos considerados innovativos desde el punto de vista financiero pueden erosionar ese papel al estar dirigidos no a llenar las funciones económicas del sistema financiero (movilizar recursos del ahorro a la inversión y compensar la información asimétrica involucrada en este proceso; evaluar la rentabilidad ajustada por riesgo de los respectivos proyectos y manejar el riesgo implicado mediante su diversificación y el monitoreo del uso de los recursos) sino para maximizar su propio beneficio trasladando el riesgo a otros sectores mediante apalancamiento y combinación no transparente de instrumentos con diversos grados de riesgo. Este es un ejemplo de divergencia de rentabilidad privada y rentabilidad social que pone de manifiesto la necesidad de regulación de la actividad empresarial privada para que ella no vaya en contravía de las posibilidades de bienestar social. En este caso particular, de las abiertas por el cumplimiento por parte del sistema financiero de su función como cerebro de la actividad económica e institucionalidad por excelencia llamada a compensar la información asimétrica (no a incrementarla como lo hacen algunas de las mencionadas innovaciones financieras), contradicción que puede minar un funcionamiento de mercados tan cruciales para el desarrollo.

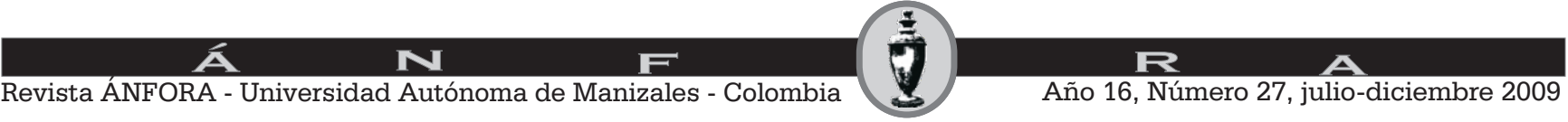


Sin la consideración de estas instancias de falla de mercado el estudiante de negocios abordará el tema de las finanzas sin una referencia a la función económica del sistema financiero, confundiendo la profundización y sofisticación financieras con fortalecimiento de su capacidad para cumplir esa función. Perderá así de vista aspectos tan importantes como los ventajas de los diversos modelos de desarrollo financiero (basado en bancos vs. basado en mercado de capitales) y las estrictas condiciones bajo las cuales el mercado de capitales puede intermediar recursos a la inversión productiva en la forma eficiente que lo asumen los análisis financieros de sus instrumentos. Desde luego se trata de que sobre la base del entendimiento de la lógica financiera de ellos se entienda también los supuestos institucionales y regulatorios que desde el punto de vista económico son necesarios para que efectivamente faciliten esa intermediación.

3) La dimensión tecnológica. Un contraste similar al que se presenta en materia financiera entre, por una parte, enormes posibilidades abiertas por las gigantescas masas de recursos (y los mecanismos para su movilización expedita posibilitados por la integración global de los mercados financieros y por el papel de las TIC en esta integración), y por otra, la imposibilidad de hacer llegar recursos financieros a agentes críticos del desarrollo, se da en el área tecnológica. Aspectos de las TIC como el Internet y las capacidades multiplicadas de almacenamiento, procesamiento y transmisión de información, han incrementado las posibilidades de difusión tecnológica (abriendo windows of opportunity for leapfrogging) que se concretan a través de redes que posibilitan la intensidad de los procesos productivos en conocimiento tecnológico y la interfase informacional características de la knowledge economy. Sin embargo el carácter acumulativo y sendero dependiente del proceso de acumulación de capacidades tecnológicas que determina que la capacidad de absorción esté determinada por las capacidades previamente acumuladas, significa que agentes que se ven marginados de esas redes por su carencia de estas capacidades enfrentan barreras crecientes en el acceso a las posibilidades abiertas por ellas. Se trata de una situación de falla de mercado por asimetrías de información que reflejan asimetrías estructurales, similar a la que se da en el área financiera: en ambos casos la ausencia de mecanismos regulatorios o cooperativos de compensación de falla de mercado conduce a la situación descrita en el dictum del evangelio de SMateo: al que tiene mucho mas le será dado y aquel que tiene poco lo poco que tiene le será quitado. 
Áreas del conocimiento en que se considere la dimensión económica de la estrategia empresarial (organización industrial), y de las condiciones financieras (economía de la financiación) y tecnológicas (economía de la innovación) de la actividad inversora central a esa estrategia le ofrecen al estudiante de negocios el entrenamiento que le permite abordar estas problemáticas centrales de estos en una perspectiva económica. La consideración de la dimensión internacional refuerza la necesidad del entrenamiento en esas áreas ya que lograr la competitividad en el nuevo sentido que ésta ha adquirido en condiciones de globalización (de integración en la globalización productiva/comercial, financiera y tecnológica) requiere un entendimiento tanto del comportamiento estratégico de sus agentes fundamentales como del carácter y la lógica que gobierna tanto las tres formas de la globalización como las sinergias entre ellas. Los correspondientes temas de desarrollo industrial y sus condiciones tecnológicas y financieras, así como su dimensión de competitividad internacional se articulan en una forma funcional a la sensibilización destacada a la dimensión social de la actividad empresarial en las discusiones de un curso sobre desarrollo económico. El cual desde luego tendrá que privilegiar, en las condiciones actuales de globalización, la consideración de los mecanismos y estrategias para integrarse competitivamente en dichas tres formas.

Finalmente, una aproximación que permite articular el abordamiento de diversos problemas en una forma integrada es su discusión en un curso de Desarrollo, o -particularmente en el caso de los Negocios Internacionales- un curso de Desarrollo y Globalización. Los problemas de organización industrial, de economía de la financiación y de economía de la innovación se pueden plantear en forma de implementar la idea de entrenar en el abordamiento y la solución de problemas empresariales desde una perspectiva económica en las discusiones sobre competitividad, financiación del desarrollo y cambio técnico que hacen parte de los aspectos macro, meso y micro del desarrollo económico. 